\title{
MODELLING DESIGN TRADE-OFFS USING A SET THEORY APPROACH: ALTERNATIVE PATHS FOR NAVIGATING TRADE-OFF SITUATIONS
}

\author{
Nickel, Jordan; \\ Hurst, Ada; \\ Duimering, P.Robert \\ University of Waterloo
}

\begin{abstract}
This paper synthesizes concepts from the design creativity and design optimization literatures to develop a conceptual descriptive model of trade-off situations in design. Using a set theory approach, a model of the design space is expanded to formalize the description of trade-offs as Pareto frontiers on this space. The modelling of design process and human biases and limitations on the structure of these design spaces explores the perceptions designers form of these design spaces. The model presented describes how altering the framing and formulation of the design space can be used to alter or bypass the original Pareto frontiers of that space, allowing trade-offs to be navigated outside of the original limitations.
\end{abstract}

Keywords: Creativity, Decision making, Design theory, Design Space, Trade-off

\section{Contact:}

Nickel, Jordan Joshua

University of Waterloo

Management Sciences

Canada

jnickel@uwaterloo.ca 


\section{INTRODUCTION}

A design trade-off is a situation where gains made in one attribute come at the cost of losses in at least one other attribute (Byggeth and Hochschorner, 2006, p. 1420). Designers routinely encounter tradeoffs in their design practice, limiting their options and forcing decisions to be made about priorities (ibid.). These decisions are typically complex, involving multiple competing criteria and stakeholders (ibid.). The field of design optimization attempts to provide optimal solutions to these problems, within a set problem structure and the limitations associated with that structure.

The design creativity literature and the history of technology present many examples of instances where innovative solutions were developed that were able to bypass perceived limitations of a problem, ranging from the simple but hidden solution to the 9-dot problem (Akin and Akin, 1996) to highly complex design problems (Crilly and Moroşanu Firth, 2019). These instances have been colloquially referred to as "eureka!" or "aha!" moments, strokes of creative genius, innovative solutions, or sudden mental insights (Akin and Akin, 1996; Chandrasekera et al., 2013). Our work connects the understanding of these sudden mental insights from the creative design literature with concepts from the design optimization and decision-making literature to model trade-off situations and the possible mechanisms to navigate and bypass trade-offs.

The approach to bypassing trade-offs in design detailed in this article has precedence in the study of innovative and creative design, such as in the TRIZ methodology and its focus on eliminating contradictions (Altshuller, 1984), or in the mechanism of adding variables to expand the design space found in Gero and Kumar's (1993) model of design as state-space search. The formulation of a design space using a set theory approach presented in this paper builds on Gero and Kumar's (1993) model by formalizing a structure to model these spaces using the concepts of parameters, membership criteria and elements from set theory (citation?).

Pareto optimality and its associated concept of Pareto frontiers were originally developed in the context of economic optimality (Ekelund and Hebert, 2007) but have since been applied to a broad variety of optimization problems, including design optimization (Gero and Kazakov, 2000; Mattson et al., 2019; Rafiq, 2000). The concept of extending or expanding the Pareto frontier to push beyond the initial limits of a problem has also been explored in the design literature (Dubois et al., 2015; Gero and Kazakov, 1999).

We build on this work to present a formally structured model capable of describing how creative design solutions navigate trade-offs. By applying a set theory approach, an underlying design space structure can be described and with it the mechanisms by which this structure permits the navigation of its boundaries. The remainder of this paper is organized into model description, limitations and future work, and conclusion. The model description is divided into four sections: describing the mathematical concepts, modelling design using these mathematical concepts, describing how human processes and limitations are captured in the model, and formalizing the trade-off situations and their navigation within the model.

\section{MODEL}

\subsection{Mathematical Underpinnings of the Design Space}

The design space, which has occasionally been divided into a problem space and a solution space in the literature (Dorst and Cross, 2001; Poon and Maher, 1997), is a mathematical representation of the set of solutions to a given design problem (Gero and Kumar, 1993). The design space model as formalized below represents an instantaneous "slice" of the solutions at a given instant in the design process, given the current problem formulation at that instant. It should be noted in advance that this model of the design space is an abstraction and not a prescriptive methodology for designers to follow directly, but may inform how designers navigate the design space when confronted with perceived trade-offs. The model presented in this section makes no assumptions about the design process and the order of actions taken by designers in practice. The formalization of design spaces allows for inferences to be drawn about the underlying structure of trade-offs and the mechanisms by which they emerge and can be navigated.

Formally, a mathematical set is defined by its elements and its membership criteria (Halmos, 1974). For the purposes of this model, the naïve set theory approach will be used for its simple and direct correlation to natural language description of sets (Halmos, 1974). In addition to the elements of the 
set and the membership criteria, one other aspect is fundamental to the application of this theory to describe design: the parameters used to characterize and differentiate elements (i.e., designs) from each other. Figure 1 below provides a visual overview of the concepts discussed in the coming sections.

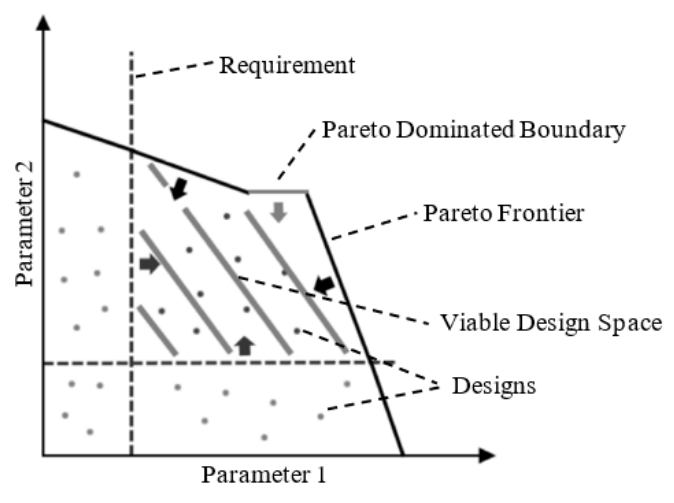

Figure 1. Diagram of design space components

\subsubsection{Elements}

The elements of the design space are simply designs, in the noun usage of the term: the output of the design process. Elements are characterized by parameters, and these parameters are what differentiates individual designs from one another. Conceptually, the elements are fully characterized instances of designs, making them distinct from design families (Shi and Gero, 2000). The inclusion of these elements into the design space is determined by the membership criteria, but designs can exist outside of the design space as shown in Figure 1. Elements that are included in the design space can also be called solutions to the design problem, as they are viable solutions to the problem as currently framed.

\subsubsection{Membership Criteria}

The membership criteria of a set determine which elements are included in the set and which elements are excluded. This determination functions as the boundaries of the design space, such as the requirements and Pareto frontiers that are shown in Figure 1.

\subsubsection{Parameters}

The last fundamental aspect of this model is the parameters used to characterize the elements. A parameter is any attribute, characteristic, or aspect that can be used to compare and contrast elements. Parameters can have different scales of measurement, ranging from nominal parameters to ratio level parameters (Stevens, 1946). They can be quantitative or qualitative, continuous or discrete; and parameters may be measured at different degrees of precision.

\subsubsection{Pareto Frontiers}

Formally, a trade-off situation can be described by the associated concepts of Pareto optimality and Pareto frontiers. Pareto optimality is a state of a system or an element where no parameter can be improved without an associated loss in at least one other parameter (Schulz et al., 2018). The Pareto frontier is the collection of states where each state exhibits Pareto optimality, or alternatively, where there exist no states which Pareto dominate it (ibid.). A state is Pareto dominated when there exists the potential for a Pareto improvement, which is a new state where gains can be made in an attribute(s) without any attribute(s) experiencing a loss (ibid.).

The Pareto concepts outlined above are a formalization of optimality that aligns with the more colloquial concept of a trade-off introduced earlier in this article. The Pareto frontier is an attribute of a design space that represents one form of boundary to the design space for a problem. It occurs due to a conflicting or antagonistic, relationship between two or more parameters. For a Pareto relationship between parameters to occur, all the parameters involved must be characterized at an ordinal level at a minimum. This is because Pareto dominance cannot occur if designs cannot be ordered in terms of desired performance with respect to the parameters in question. Each Pareto frontier is associated directly with the design space that it bounds and changing either the membership criteria or the parameters of that design space can potentially alter or eliminate these Pareto frontiers. 


\subsection{Modeling Design using the Design Space}

\subsubsection{Membership Criteria}

In the context of the design space, the membership criteria determine the viability of a design as a solution to the problem at hand. Viability in this model is defined to be the logical status (e.g., true versus false) of a design instance relative to the attainment of the goal state(s) of the design problem as it is currently formulated.

The boundaries of the design space can originate from a variety of sources, as anything that affects the viability of a design as a solution imposes a boundary. Examples include but are not limited to boundaries imposed by natural laws and constraints; the requirements, constraints and criteria of the design brief; regulations, laws and societal expectations; project resources allocated for design, production, distribution, recovery/disposal, etc.; and limits of currently available technology. Boundaries to viability can also be imposed and altered by the decisions the designer makes during the design process, such as the selection of structures or approaches to solving the problem, or the negotiation of previously imposed limits.

The intersection of the boundaries created by the membership criteria is the viable design space and contains all of the viable design solutions given those membership criteria, which is shown by the cross hatched area of Figure 1. Furthermore, membership criteria in design can have differing degrees of negotiability (Goel and Pirolli, 1992), from entirely non-negotiable constraints like the speed of light, through constraints like legal regulations that are non-negotiable on the scale of a design project, to completely negotiable and arbitrarily imposed bounds like colour preferences.

\subsubsection{Parameters}

Design goals often begin with ill-defined, common-language descriptions of the intended functionality of the design outcomes (Goel and Pirolli, 1992). This model uses parameters to operationalize the goal state(s) of the design problem, to differentiate individual elements from one another through comparison of the elements' values along the parameter, and to assign preference order along that parameter.

A goal state can potentially be operationalized using different parameters and membership criteria, resulting in different design spaces. For example, the goal state of "move people efficiently from point A to point B" may be operationalized using the parameters speed and fuel efficiency; or it may be operationalized using the parameters average journey time and passenger capacity. The first operationalization may direct a designer towards more efficient single occupancy vehicles as a solution, while the latter may hint at multimodal mass transit.

While there exists a potentially infinite number of parameters that could characterize a design space, the nature of human designers and their limits reduces this to a much smaller subset of parameters. This model captures this as the designers making parameters "explicit", through their conscious consideration of a parameter at some point during the design process. Parameters can also have their explicit status discarded by the designer if they are found to be irrelevant to the design problem and its goal(s). Both processes affect the dimensionality of the design space, which is the number of explicit parameters being used to characterize the design space at any time. The parameters made explicit to characterize a problem by a designer are subjective based on their understanding of the problem, and different designers may use different parameters to describe the same problem.

An example of a parameter being made explicit is a designer considering whether the colour of a car may influence the top speed. In this scenario, the designer makes the parameter "colour" an explicit parameter of a design space with a goal state operationalized along the "speed" parameter, increasing the design space dimensionality by 1 . If the designer then decides or determines that the colour of the car has no relationship to its speed, then they may decide to either discard the colour parameter from consideration or maintain it as a purely nominal parameter with no bounds or ordinality.

The introduction of membership criteria can induce the addition of explicit parameters. Conversely, making a parameter explicit can also drive the consideration of new membership criteria. Returning to the previous car example, an example of membership criteria inducing further explicit parameters could be the marketing department providing information on the sales figures of different coloured cars and imposing a goal for minimum sales for the new car being designed. This sales goal may lead the designer to the explicit incorporation of colour as a parameter, with bounds and ordinality being imposed on that parameter by the previous sales data. An example of the converse may be that 
considering the ergonomics as an explicit parameter when designing a pen may lead to the setting of ergonomics requirements.

Parameters can also be associated with other parameters, and new parameters may be introduced and made explicit by their association with other parameters, by the designer proposing a solution concept, or through the evaluation and consideration of a solution concept. For example, a qualitative parameter that characterizes the form/operating principle of a writing implement may have discrete values of pen, pencil, chalk, etc. Each of these may introduce associated parameters, for example selecting the value of pen would introduce parameters related to ink colour or viscosity, while a pencil would introduce parameters related to the graphite.

Parameters can also have hierarchical structures, where parameters can be decomposed into several sub-parameters, or several parameters can be subsumed into a higher level, more abstract parameter. An example of decomposition could be a parameter of mass being divided into density, and physical dimensions.

Covariance and causal relationships between parameters can also exist, with changes in the value of one parameter affecting the possible values of another parameter. In fact, antagonistic relationships between parameters are responsible for trade-offs in this model.

\subsubsection{Pareto Frontiers}

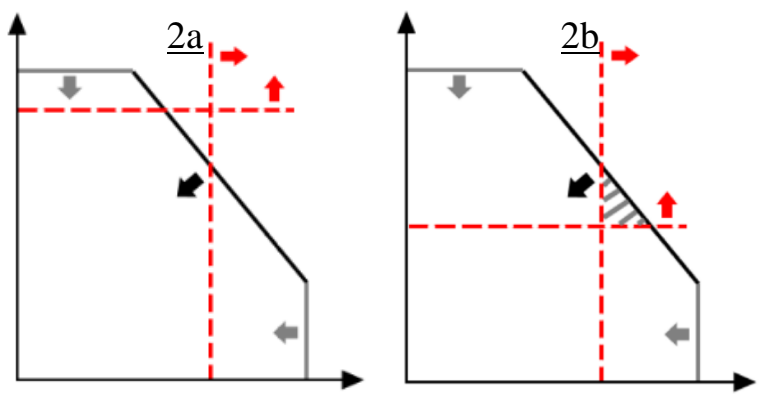

Figure 2a. A Null design space and $2 b$. Relaxing a requirement

The set formed by the intersection of a Pareto frontier and the membership criteria representing the goal states along a set of parameters can drive the formation of a null design space, where no viable solutions exist, as in Figure 2a above. A typical design response to this situation is the relaxation (i.e. negotiation) of one of these boundaries or goals such as in Figure $2 b$, although this is generally viewed as a suboptimal approach (Gero and Kumar, 1993). Pareto frontiers can also occur in a design space with a non-zero viable region, for instance, if there are designs that meet all minimum requirements or goals of a project, but the designers are not satisfied with those solutions and want to design a better solution (ibid.).

\subsection{The Influence of Human Designers on Design Spaces}

At this point it should be reiterated that the design space representation outlined above is a descriptive abstraction of the design situation that will be used to illustrate the potential approaches to navigating trade-off situations. The design space itself is typically too complex to characterize fully at any given instant due to the large number of viable solutions, and it evolves as the design process proceeds. The introduction of Pareto optimality and frontiers should not be taken as a recommendation that designers precisely characterize the design space to locate these frontiers. Instead, the design space representation should drive inferences that will aid designers in their metacognitive awareness of what a trade-off entails and how it came to be.

Given the complexity and prohibitive resource cost of perfectly characterizing the design space, it is important to consider how human factors and limitations may affect how designers cognitively model and interact with their design spaces in practice. Designers' limited cognitive models of the structure and properties of their design spaces impose both conscious and subconscious limitations on the design solutions under consideration.

\subsubsection{Cognitive Information Processing Limits}

Human cognition has limited information processing capacity (Miller, 1956; Simon, 1997), which limits the capability of designers to model the entirety of the design space, and the decisions they 
make based on those models. Even considering only explicit parameters, as detailed above, complex design situations often involve too many explicit parameters for human designers to consider simultaneously (Miller, 1956).

Instead, it is proposed that human designers will operate with a set of active parameters, with parameters shifting in and out of active cognition as attention is paid to them. These active parameters form a lens through which the designer perceives the structure of the design space, and this can influence how they model conflicts between parameters.

The phenomenon of sudden mental insight, colloquially called the "aha!" moment, has been described as a problem reframing or restructuring (Akin and Akin, 1996). As the designer's perception shifts, these changes of focus drive new insight into problem structures and dramatically effect the final design solution (Chandrasekera et al., 2013; Suwa and Tversky, 1997). This phenomenon is modeled as a shift in the set of active parameters the designer is using to frame the problem.

\subsubsection{Fidelity of Cognitive Model}

Because human designers have limited information processing capacity (Simon, 1997), they rely on heuristics, assumptions, previous experience, and other tools to simplify their cognitive models of the design space. Such simplifications may introduce biases and errors relative to the objective design situation, and presuppositions about the potential range of viable solutions in a given design space. For example, a designer might have presuppositions about what solutions may be viable for a problem based on previous experience, heuristics, training, or other factors (Crilly, 2015). These presupposed limits to viability represent the boundaries that the designer believes are on the design space, and these limits may or may not represent the actual boundaries of viability.

These presupposed limits have a benefit of restricting the design space to be more manageable for the designer, but they may also artificially restrict the design space and eliminate possible solutions that outperform the designer's preconceived limits. In the context of trade-offs, this can be represented by the designer assuming a Pareto frontier exists at particular combinations of values along the intersection of the parameters in conflict, while the actual Pareto frontier exists at different values of those parameters, potentially Pareto dominating the perceived frontier.

\subsubsection{Knowledge Base and Situatedness}

Incomplete or imperfect knowledge, either at the individual or at the societal level, affects the changes a designer can make consciously to the design space. If designers do not have access to the knowledge necessary to conceive of certain viable solutions, then those solutions are effectively placed outside the bounds of viability of that particular designer's design space until they attain that knowledge (Hatchuel and Weil, 2009). Designers with a broader or more relevant knowledge base may be able to conceive of these solutions, thereby including them in their design space. This notion has been called situated designing, where designers' unique experiences leads to different results for a given design process (Gero and Kannengiesser, 2007). In effect, this introduces variability of design spaces for different people approaching the same problem with the same framing.

\subsubsection{Design Process in Practice}

Literature on design practice shows that designers do not typically perform an exhaustive search of all viable designs. Instead, the typical design process involves a seed idea that is then developed into a family of designs by iterative changes (Crilly, 2019a; Hatchuel and Weil, 2009). Furthermore, these iterative changes include series of arbitrary decisions that initially pass with their importance unnoticed, but limit the design space (Crilly, 2019b).

Within the context of the design space model, these decisions have the effect of imposing further boundaries (membership criteria) on the design space, which reduces the size of the viable design space. Additionally, the dimensionality of the space may also be reduced through these decisions collapsing parameters onto single values by the designer "locking in" a value on that parameter, either permanently or temporarily. Indeed, the process of design could be approximated as the progressive reduction of the design space until a single solution is selected at the end. This reduction can be reversed, redirected or reset by problem reformulation and the coevolution of the problem with the development of solutions (Dorst and Cross, 2001), but at the end of a design process a solution is selected from the design space or the design problem remains unsolved. 


\subsection{Navigating Trade-offs by Manipulating the Design Space}

The core of this paper's argument is that there exist forms of trade-offs that are inherent only to the design space the designer is currently operating in. By establishing this link, it can then be proposed that for these trade-offs an alternative method of navigating them exists: the manipulation or alteration of the design space itself. This differs from the approach to trade-offs found in the design optimization and decision-making literature, which focusses on how to select the best option within a set design space (Schulz et al., 2018).

By altering the design space, the designer has the capability to alter the Pareto frontier location, effectively manipulating the trade-off situation itself. Manipulating and altering the design space can take on one of two forms based on the formalization of the design space as a mathematical set: either altering the set membership criteria or the parameters used to characterize the elements.

Altering the membership criteria for the set can take a variety of forms, as many things can contribute to the determination of which solutions are viable. Negotiating requirements and imposed constraints, improving the technological capabilities, and adjusting the problem framing are all possible mechanisms for altering the set membership criteria.

The parameters of the design space can be influenced in multiple ways as well. For example, parameters can be added to the design space by being made explicit; they can be combined, decomposed, or substituted into alternative representations; they can be collapsed by "locking in" decisions, or expanded again by revisiting a previous decision.

With respect to navigating trade-offs, both approaches to manipulating the design space have merit but the mechanisms used to manipulate the design space vary in effectiveness and desirability. For example, while negotiating a lower requirement for an attribute like an emissions target (or even dropping that attribute from consideration altogether) may make it possible to navigate a trade-off between that target and the cost of the design successfully, most would view this as sacrificing one of the goals of the design.

Of particular interest are mechanisms that allow designers to "bypass" the Pareto frontier in the original design space, without negotiating or sacrificing the goals of the design. Mechanisms to do this vary based on what factors are driving the Pareto frontier's existence, but examples include altering the dimensionality of the design space or changing the operationalization used for a goal. In design terms, this may include making another parameter explicit that influences the values of parameters involved in the trade-off.

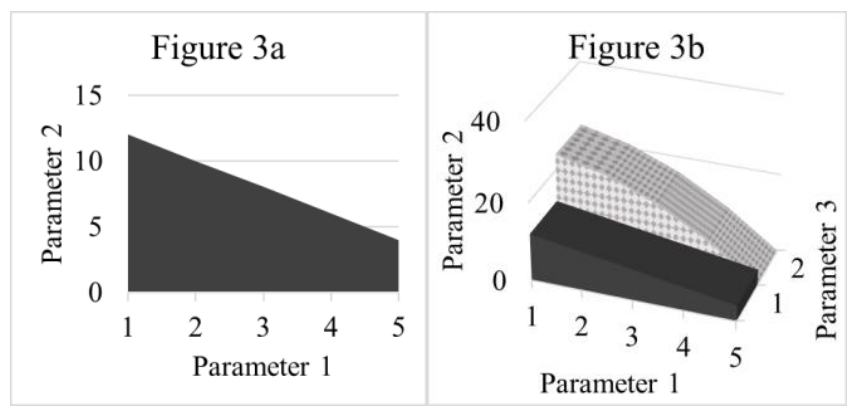

Figure 3. Design space expansion through the introduction of a third, discrete, parameter

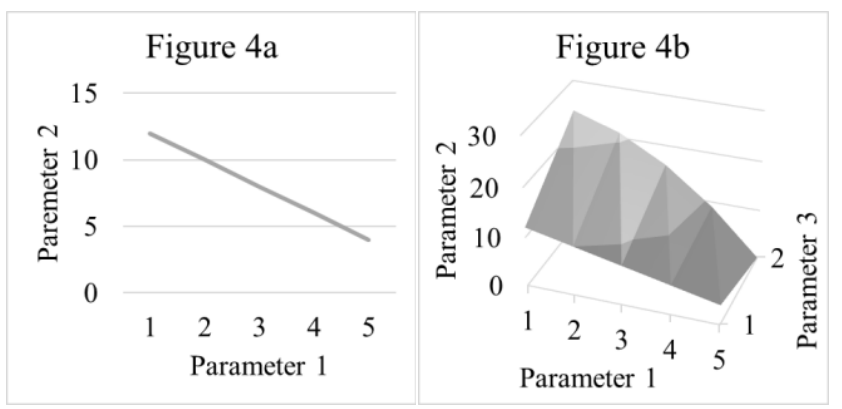

Figure 4. Design space expansion through the introduction of a third, continuous, parameter 
For illustration of how this facilitates bypassing a trade-off see the design spaces in Figures 3 and 4 above, which show the addition of a discrete parameter and the addition of a continuous parameter respectively. Figures $3 \mathrm{a}$ and $4 \mathrm{a}$ show a design space with two parameters, which are directly antagonistic. As the parameter values "increase" along parameter 1, they "decrease" along parameter 2. If the designer wants a solution that has a value of 3 for parameter 1 and a value of 10 for parameter 2 , that is not possible in the current design space. However, if the designer makes explicit a third parameter that has a relationship with the two original parameters, as in Figures $3 b$ and $4 b$, then it may become possible to create designs with alternative values on the third parameter and obtain the target values for parameters 1 and 2 .

Consider a simplified example where a designer encounters a trade-off between the strength of a steel beam and the weight of that beam. A potential mechanism this designer could use to bypass this trade-off as it currently exists is making explicit additional parameters that could affect the viability of the design, such as the material of the beam or the cross-sectional shape of the beam. As the designer explores different "levels" of these new parameters, the relationship between the original parameters of interest (strength \& weight) changes. The selection of a different material, or a differently shaped cross-section, may allow the designer to improve the strength of the beam relative to the original trade-off without increasing its weight. By altering the dimensionality of the design space in this example, it becomes possible to travel along the third dimension to navigate beyond a Pareto frontier that was previously restricting the design space. While the example presented above was restricted to 3 dimensions for easier graphical illustration, dimensionality changes can alter the size and shape of the viable design space at any level of dimensionality.

\section{LIMITATIONS \& FUTURE WORK}

There are several important limitations to the conceptual model introduced in this paper and the conclusions drawn from it. Most pressing is that the model was developed through the synthesis of existing literature and design theories and has been illustrated through hypothetical examples. To further validate the theory, empirical investigation is needed on the structure of trade-offs and the approaches used by designers to navigate them in practice. To this effect, the authors are presently engaged in a qualitative interview study to investigate designers' perceptions and models of trade-offs they have encountered and successfully navigated. Another limitation of the model as currently presented is that it has a limited treatment of the temporal evolution of design spaces. Further work is needed to characterize how design spaces evolve over time, as the model presented in this work is primarily described in static "slices" at instantaneous points in the design process. For example, studying how designers arrive at the parameters they make explicit and the factors that influence this process would help clarify how designers alter their design spaces over time.

Drawing on other design theories like C-K Theory (Hatchuel and Weil, 2009) and Shai's Infused Design (Shai and Reich, 2004) may also inform how designers navigate and create their design spaces. For example, the operators from C-K Theory (Hatchuel and Weil, 2009) provide insight into the process by which designers navigate their design space. For example, operations moving from the concept space $(\mathrm{C})$ to the knowledge space $(\mathrm{K})$ would increase the fidelity of the designer's conceptual model to the underlaying structure of the design space in that instant, while $\mathrm{C}->\mathrm{C}$ operators may explain how designers identify parameters and map elements of the design space. Infused Design (Shai and Reich, 2004) theory hints at the benefits of collaboration for expanding and exploring the design model. By using Infused Design's combinatorial representation methods to improve crossdisciplinary communication, new parameters that are common in one domain can be introduced to another domain with a reasonable prediction of how that parameter would affect the design space, rather than a simply making intuitive or educated guesses at which parameters to add.

The treatment given to this model in this paper was also limited by its presentation of design as the process of individual designers. This was done intentionally to facilitate the communication of the fundamentals of the model, but it discounts the influences of social dynamics on the design process. Examples of these include pressures imposed by management to limit design space exploration to accelerate the timeline of the design, or the degree of similarity between mental models of the design space across team members. Social dynamics also have potentially positive influences on the design process, such as the diversity of background and knowledge helping to identify novel and relevant parameters to investigate, or different perspectives helping to break-out of the current perceptual 
problem structure. Further exploration of these factors will help the model more accurately describe the nature of design in practice, where these social dynamics play a critical role.

An expansion of this work could also investigate the potential of using this model or derivatives from it as interventions for improving design practice. Studying whether awareness of design space structure and the mechanisms to manipulate them improves designer's ability to navigate trade-offs successfully would connect this conceptual work to design practice.

\section{CONCLUSION}

The conceptual model presented in this article describes design trade-offs in a formal sense using the set theory construct of a design space. This structure harmonizes the phenomena noted in the creative design literature with the structure of trade-offs as described by the design optimization literature. It also illustrates that the approach of immediately attempting to optimize in the face of trade-offs neglects the alternative approaches present in the creative design literature. This behaviour has consequences, particularly when goals and parameters of broader and externalized import like sustainability or social impact come into conflict with traditional parameters like cost and safety. The navigation method suggested by this article offers an alternative approach based on principles of creative design that has the potential to prevent or mitigate the sacrifices designers need to make when faced with these conflicts.

\section{REFERENCES}

Akin, Ö. and Akin, C. (1996), "Frames of reference in architectural design: Analysing the hyperacclamation (Ah-a-!)", Design Studies, Vol. 17 No. 4, pp. 341-361, https://doi.org/10.1016/S0142-694X(96)00024-5.

Altshuller, G.S. (1984), Creativity as an Exact Science: The Theory of the Solution of Inventive Problems, Gordon and Breach Science Publishers.

Byggeth, S. and Hochschorner, E. (2006), "Handling trade-offs in Ecodesign tools for sustainable product development and procurement", Journal of Cleaner Production, Vol. 14 No. 15-16, pp. 1420-1430, https://doi.org/10.1016/j.jclepro.2005.03.024.

Chandrasekera, T., Vo, N. and D'Souza, N. (2013), “The effect of subliminal suggestions on Sudden Moments of Inspiration (SMI) in the design process", Design Studies, Vol. 34 No. 2, pp. 193-215, https://doi.org/10.1016/j.destud.2012.09.002.

Crilly, N. (2015), "Fixation and creativity in concept development: The attitudes and practices of expert designers", Design Studies, Vol. 38, pp. 54-91, https://doi.org/10.1016/j.destud.2015.01.002.

Crilly, N. (2019a), "Creativity and fixation in the real world: A literature review of case study research", Design Studies, Vol. 64, pp. 154-168, https://doi.org/10.1016/j.destud.2019.07.002.

Crilly, N. (2019b), "Methodological diversity and theoretical integration: Research in design fixation as an example of fixation in research design?", Design Studies, Vol. 65, pp. 78-106, https://doi.org/10.1016/j.destud.2019.10.006.

Crilly, N. and Moroşanu Firth, R. (2019), "Creativity and fixation in the real world: Three case studies of invention, design and innovation”, Design Studies, Vol. 64, pp. 169-212, https://doi.org/10.1016/j.destud.2019.07.003.

Dorst, K. and Cross, N. (2001), "Creativity in the design process: Co-evolution of problem-solution", Design Studies, Vol. 22 No. 5, pp. 425-437, https://doi.org/10.1016/S0142-694X(01)00009-6.

Dubois, S., Lin, L., De Guio, R. and Rasovska, I. (2015), "From simulation to invention, beyond the paretofrontier", Proceedings of the 20th International Conference on Engineering Design (ICED 15), Vol. 6, Milan, IT.

Ekelund, R.B. and Hebert, R.F. (2007), A History of Economic Theory and Method, 5th ed., Waveland.

Gero, J.S. and Kannengiesser, U. (2007), "An ontology of situated design teams", Artificial Intelligence for Engineering Design, Analysis and Manufacturing: AIEDAM, Vol. 21 No. 3, pp. 295-308, https://doi.org/10.1017/S0890060407000297.

Gero, J.S. and Kazakov, V. (1999), “An Interpolation/Extrapolation Process For Creative Designing”, Computers in Building, pp. 263-274, https://doi.org/10.1007/978-1-4615-5047-1_17.

Gero, J.S. and Kazakov, V. (2000), "Adaptive enlargement of state spaces in evolutionary designing”, Artificial Intelligence for Engineering Design, Analysis and Manufacturing, Vol. 14 No. 1, pp. 31-38, https://doi.org/10.1017/S0890060400141034.

Gero, J.S. and Kumar, B. (1993), "Expanding design spaces through new design variables", Design Studies, Vol. 14 No. 2, pp. 210-221, https://doi.org/10.1016/0142-694X(93)80048-H.

Goel, V. and Pirolli, P. (1992), "The structure of design problem spaces", Cognitive Science, Vol. 16 No. 3, pp. 395-429, https://doi.org/10.1016/0364-0213(92)90038-V. 
Halmos, P.R. (1974), Naive Set Theory, edited by Axler, S., Gehring, F.W. and Ribet, K.A., Springer, New York, https://doi.org/10.1007/978-1-4757-1645-0.

Hatchuel, A. and Weil, B. (2009), “C-K design theory: An advanced formulation”, Research in Engineering Design, Vol. 19, pp. 181-192, https://doi.org/10.1007/s00163-008-0043-4.

Mattson, C.A., Pack, A.T., Lofthouse, V. and Bhamra, T. (2019), 'Using a Product's Sustainability Space as a Design Exploration Tool”, Design Science, Vol. 5, pp. 1-34, https://doi.org/10.1017/dsj.2018.6.

Miller, G.A. (1956), "The Magical Number Seven, Plus or Minus Two: Some Limits on Our Capacity for Processing Information", The Psychological Review, Vol. 63 No. 2, pp. 81-97, https://doi.org/10.1037/h0043158.

Poon, J. and Maher, M. Lou. (1997), "Co-evolution and emergence in design”, Artificial Intelligence in Engineering, Vol. 11 No. 3, pp. 319-327, https://doi.org/10.1016/S0954-1810(96)00047-7.

Rafiq, M.Y. (2000), "Importance of Pareto optimum solutions in making informed decisions in engineering design”, Computing in Civil and Building Engineering, Stanford, California, pp. 1325-1333, https://doi.org/10.1061/40513(279)173.

Schulz, A., Wang, H., Grinspun, E., Solomon, J. and Matusik, W. (2018), "Interactive exploration of design trade-offs", ACM Transactions on Graphics, Vol. 37 No. 4, https://doi.org/10.1145/3197517.3201385.

Shai, O. and Reich, Y. (2004), "Infused design. I. Theory”, Research in Engineering Design, Vol. 15 No. 2 , pp. 93-107, https://doi.org/10.1007/s00163-004-0047-7.

Shi, X. and Gero, J.S. (2000), "Design families and design individuals", Engineering with Computers, Vol. 16, pp. 253-263, https://doi.org/10.1007/s003660070008.

Simon, H.A. (1997), "Bounded Rationality", Models of Bounded Rationality: Emperically Grounded Economic Reason, Vol. 3, The MIT Press, Cambridge, MA, https://doi.org/10.7551/mitpress/4711.001.0001.

Stevens, S.S. (1946), “On the Theory of Scales of Measurement”, Science, Vol. 103 No. 2684, pp. 677-680, https://doi.org/10.1126/science.103.2684.677.

Suwa, M. and Tversky, B. (1997), "What do architects and students perceive in their design sketches? A protocol analysis”, Design Studies, Vol. 18 No. 4, pp. 385-403, https://doi.org/10.1016/s0142-694x(97)00008-2. 